\title{
Intradiscal Therapy and Transforaminal Endoscopic Decompression: Opportunities and Challenges for the Future
}

\section{Anthony $\mathrm{T}$ Yeung ${ }^{*}$}

University of New Mexico, School of Medicine, Albuquerque, New Mexico, USA

*Corresponding author: Anthony T Yeung, University of New Mexico, School of Medicine, Albuquerque, New Mexico, USA, Tel: (602) 944-2900; E-mail: ayeung@sciatica.com

Rec date: Sep 21, 2016; Acc date: Sep 22, 2016; Pub date: Sep 26, 2016

Copyright: (C) 2016 Yeung AT. This is an open-access article distributed under the terms of the Creative Commons Attribution License, which permits unrestricted use, distribution, and reproduction in any medium, provided the original author and source are credited.

\section{Introduction}

Fusion is a common surgical goal by traditional surgeons to eliminate debilitating back pain and sciatica, especially when nonsurgical or simple decompressions fail. Spine surgeons trained to perform fusions, with the ultimate goal of addressing deformity and instability, leave a big gap of treatment options between surgery and pain management procedures. With no training in endoscopic surgery, however, both surgeons and interventionalists struggle to battle a crisis of escalating cost and affordability when using fusion as well as ineffective long-term results of pain management as the ultimate solution.

Intradiscal techniques in the literature, validated by level I studies for chymopapain, culminating with endoscopic decompression, ablation and irrigation, now support Selective Endoscopic Discectomy $^{\text {tw }}$ with thermal annuloplasty for discogenic back pain. This promising surgical intervention for discogenic back pain is reported in the literature. Selective Endoscopic $\left(\right.$ Discectomy $\left.{ }^{\mathrm{rm}}\right)\left(\mathrm{SED}^{\mathrm{rx}}\right)$ is a visualized intradiscal procedure that incorporates endoscopic discectomy with intra-operative evocative chromo discography", and thermal annuloplasty [1-5].

Fernstrom coined the term "discogenic pain" in 1960 [6] through experimental in vivo stimulation of the annulus fibrosus. This established the association between annulus stimulation and subjective pain perception. Histologically, the end organ neural sensors are located in the outer layers of the annulus, and the juxta- endplate region [7-9]. The nucleus pulposus and its metabolic by products are known contact irritants to nerve tissue. It is hypothesized that the degenerative process and micro trauma lead to fissuring of the annulus fibrosus and defects in the endplates which bring the nerves into chronic contact with the cytokines from the degenerating nucleus. Defects in the annulus create an inflammatory response and ingrowth of granulation tissue, [10] new vessels, and new nerve endings. Chronic exposure of the acidic irritants in the annular defects is hypothesized to be the local pain sensitization pathway that leads too chronic lumbar discogenic pain (CLDP).

Chronic lumbar discogenic pain is a difficult condition to treat, as its pathogenesis is multifactorial and only partially understood. Recent studies suggest that debilitating pain involves irritation of the dorsal root ganglion. Non-operative therapeutic methods often fail to achieve sufficient pain relief because chemical irritants emanate from the degenerating disc. Injection therapies with epidural steroids are good at relieving radiculitis, but are less successful at helping low back pain. Surgical options vary greatly, ranging from disc decompression to $360^{\circ}$ fusion.
The morbidity, however, associated with the fusion technique, while better accepted and more thoroughly studied, is significant when considering only $65 \%$ to $80 \%$ of patients obtain satisfactory clinical results [11-17]. The morbidity of the procedure often creates more problems when the procedure fails.

Discectomy is a surgical treatment procedure validated by the SPORT Study. When discogenic pain progresses to disc herniation caused by failure of the annulus fibrosis to contain the nucleus, radiculopathy may occur. Minimally Invasive surgical treatment options were later developed, notably disc decompression performed transforaminally through Kambin's triangle. Posterolateral Selective Endoscopic Discectomy ${ }^{\text {mi }}$ (SED) and radiofrequency (RF) thermal annuloplasty is a percutaneous endoscopic technique developed by Yeung, also referred to in the literature as PELD.

A 1997 IRB approved study of endoscopic spine surgery for painful degenerative conditions of the lumbar spine provided opportunities to study endoscopic intradiscal therapy. Intradiscal decompression with thermal annuloplasty has evolved to include decompression of the foramen that has successfully treated foraminal and spinal stenosis in an aging spine. Both intradiscal therapy and foraminoplasty have offered effective treatment for common back pain that can escalate into debilitating pain in some patients. This includes failed back surgery syndrome, a devastating consequence of failed traditional spine surgery. Over 10,000 endoscopic procedures documented in a personal database have made the transforaminal approach to the lumbar spine a versatile approach for a wide variety of painful lumbar conditions. The surgical technique, starting in the disc as a primary pain generator, known as the YESS $^{\text {rix }}$ philosophy and surgical technique, is aided by improved instrumentation, scopes, evolving techniques, and outcome studies using outcome method criteria such as MacNab criteria, VAS, ODI, and patient satisfaction questionnaires.

The procedures of intradiscal therapy and foraminoplasty have evolved and culminated in predictable clinical results with minimal surgical morbidity for highly experienced endoscopic surgeons. This is backed by recent peer reviewed literature, and with multiple publications increasing since 2000. Some reviews from this study base span over 10 years' follow-up for such conditions as spondylolisthesis. There are no studies that have opposed the technique as not effective.

The current trend in MIS endoscopic spine is surgical procedures with a focus on intradiscal therapy. This includes Pilot studies on intradiscal augmentation that are CE marked, featuring intradiscal augmentation implants with hydrogel sticks that can be inserted with a thin 18-gauge needle. Preliminary results demonstrate high efficacy rate with few surgical complications. Biologics to heal, slow degeneration, or even regenerate injured intervertebral discs is becoming a viable step towards that goal. 
Although lateral recess decompression has been performed with mechanical instruments since 1993, its use was limited in favor of more traditional translaminar decompression. The lack of good mechanical endoscopic tools forced early adopters to rely too much on lasers for decompression. New endoscopic instruments, many custommade such as endoscopic kerrison rongeurs, facilitated by automated burrs have helped endoscopic decompression evolve further. Recently, articulated burrs made it a routine procedure for decompression of the lateral and subarticular recess that can also reach the inlet of the lateral recess to the exit zone not accessible with translaminar decompression. This is accomplished without destabilizing the spinal segment. Visualization and decompression and ablation of painful pathoanatomy, documented in a patient awake under only local anesthesia with minimal sedation serves as the surgically based evidence. Level I evidence for endoscopic spine surgery under local is difficult and if not impossible for blinded comparison, so other evidence such as direct visualization of the surgical treatment of the pain generator should be substituted for evidence based scientific validation.

In conclusion, intradiscal therapy, combined with transforaminal endoscopic decompression is effective for chronic back pain and sciatica by decompressing the patho-anatomy in the disc, and in the axilla, called the "hidden zone of Mac Nab". Each approach is time sensitive. First focus on the disc as a pain generator, but extend the technique to foraminoplasty. Spinal anatomy is altered in a naturally aging spine. By focusing on the disc, validated by level I chymopapain studies, future considerations should include intradiscal nucleus augmentation as a means to delay the effects of the aging process. The current focus on biologics to at least delay, even if not able to reverse the effects of an aging process, bears investigation.

The percutaneous foraminoplasty decompression approach avoids going through the previous translaminar surgical sites for failed back surgery syndrome. It does not "burn bridges" for a more conventional approach, and, if the approach is used as the first alternative to fusion, it will mitigate the need for fusion for $30-70 \%$ from Yeung's review of his spondylolisthesis patients with over 10-year follow-up of patients who first opt for this less invasive philosophy and technique after nonsurgical treatment fails.

Transforaminal Lateral Recess Endoscopic decompression is a true minimally invasive procedure performed under local anesthesia. It is predictably successful when transforaminal epidural blocks provide excellent pain relief. Endoscopic surgical techniques will open the door for cost effective surgical solutions for painful degenerative conditions of the lumbar spine, warranting further study and adoption by surgeons trained adequately in this technique.

\section{References}

1. Yeung AT, Gore SA (2001) Evolving methodology in treating discogenic back pain by Selective Endoscopic Discectomy (SED). Journal of Minimally Invasive Spinal Techniques 1: 8-16.
2. Tsou PM, Alan Yeung C, Yeung AT (2004) Postero-lateral transforaminal selective endoscopic discectomy and thermal annuloplasty for chronic lumbar discogenic pain. The Spine Journal. 4(5): 564-573.

3. Yeung AT (2000) The role of provocative discography in endoscopic disc surgery, in the practice of minimally invasive spinal techniques. M.H. Savitz, J. Chiu, and A.T. Yeung, (eds). AAMISMS Education LLC 31-236.

4. Bini W, Yeung AT, Calatayud V, Chaaban A, Seferlis T (2002) The role of provocative discography in minimally invasive selective endoscopic discectomy. Neurocirugia (Astur) 13:27-31.

5. Yeung AT (2001) Intradiscal thermal therapy for discogenic low back pain, in The practice of minimally invasive spinal techniques. Savitz $\mathrm{MH}$, Chiu J, Yeung AT, (eds). J Minimally Invasive Spine Tech 1: 8-16.

6. Yeung AT, Yeung CA (2006) Microtherapy in low back pain. Mayer HM (ed): Minimally invasive spine surgery (2ndedn) Berlin Heidelberg, Germany Springer Verlag p: 267-277.

7. Fernstrom U (1960) A discographical study of ruptured lumbar intervertebral discs. Acta Chir Scand 258: 1-60.

8. Aprill CN (1996) Diagnostic disc injection, in the adult spine: Principles and practice, Frymoyer J.W. (ed.) Lippincott - Raven Publishers, Philadelphia p: 539-562.

9. Coppes MH, Marani E, Thomeer RT, Groen GJ (1997) Innervation of "painful" lumbar discs. Spine 22: 2342-2349.

10. Fras C, Kravetz P, Mody DR, Heggeness MH (2003) Substance pcontaining nerves within the human vertebral body. An immunohistochemical study of the basivertebral nerve. Spine J. 3: 63-67.

11. Kauppila LI (1995) Ingrowth of blood vessels in disc degeneration. Angiographic and histological studies of cadaveric spines. J Bone Joint Surg Am 77: 26-31.

12. Brantigan JW, Neidre A, Toohey JS (2000) The lumbar I/F cage for posterior lumbar interbody fusion and the variable pedicle screw placement system: Two-year results from a Food and Drug Administration investigational device exemption clinical trial. Spine 25: 1437-1446.

13. Kuslich SD, Danielson G, Dowdle JD, Sherman J, Fredrickson B, et al. (2000) Four-year follow-up results of lumbar spine arthrodesis using the Bagby and Kuslich lumbar fusion cage. Spine 25: 2656-2662.

14. McAfee PC (1999) Interbody fusion cages in reconstructive operations on the spine. J Bone Joint Surg Am 81: 859-880.

15. Ray CD (1997) Threaded titanium cages for lumbar interbody fusions. Spine 22: 667-679.

16. Regan JJ, Yuan H, McAfee PC (1999) Laparoscopic fusion of the lumbar spine: minimally invasive spine surgery. A prospective multicenter study evaluating open and laparoscopic lumbar fusion. Spine 24: 402-411.

17. Bono CM, Lee CK (2004) A critical analysis of trends in fusion for degenerative disc disease over the past 20 years: Influence of technique on fusion rate and clinical outcome. Spine J. 29(4): 455-463. 